\title{
FISIOTERAPIA EM GRUPO NA FORMA DE CIRCUIT TRAINING - UM MODELO FISIOTERAPÊUTICO PARA PARKINSONIANOS
}

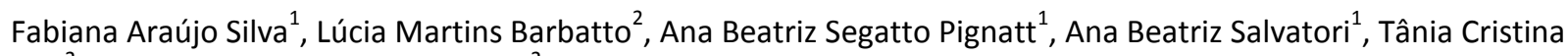
Bofi $^{2}$, Augusto Cesinando de Carvalho ${ }^{2}$.

Universidade Estadual Paulista-UNESP, ${ }^{1}$ Curso de Pós-graduação, ${ }^{2}$ Departamento de Fisioterapia, Presidente Prudente, SP. e-mail: augustocesinando@gmail.com

\section{RESUMO}

O objetivo da reabilitação após o diagnóstico da Doença de Parkinson é prevenir a progressão das desordens motoras e melhorar o desempenho funcional de parkinsonianos. A Fisioterapia em Grupo no formato de Circuit Training (FGCT) utiliza exercícios funcionais específicos com foco na repetição e na progressão contínua de exercícios em um número de estações de trabalho organizadas em um formato de circuito, para melhorar a capacidade funcional. O objetivo deste estudo é apresentar as atividades terapêuticas da FGCT e características motoras funcionais dos participantes. Participaram do estudo 14 pacientes. Este trabalho indica que a FGCT é uma terapia segura, viável e aplicável para pacientes com Doença de Parkinson, além disso, pode-se inferir que é um ambiente para o parkinsoniano realizar exercícios para prevenir a progressão das suas deficiências, diminuir a inatividade física e se adaptar às limitações.

Palavras-chave: fisioterapia, doença de Parkinson.

\section{GROUP PHYSIOTHERAPY IN THE FORM OF CIRCUIT TRAINING - A PHYSIOTHERAPY MODEL FOR PARKINSONIANS}

\section{ABSTRACT}

The aim of rehabilitation after diagnosis of Parkinson's disease (PD) is to prevent the progression of motor disorders and improve functional performance of PD. The Group of Physiotherapy in the form of Circuit Training (GPCT) uses specific functional exercises focusing on repetition and continuous progression exercises in a number of workstations arranged in a circuit format, to improve functional capacity. The aim of this study is to present the therapeutic activities of GPCT motor and functional characteristics of attendees. It participated in the study 14 patients. This work indicates that the GPCT is a safe, feasible and therapy for patients with Parkinson's disease, moreover, it can be inferred that is an environment for Parkinsonian drilled to prevent the progression of their disabilities, decrease physical inactivity and adapt to limitations.

Keywords: physiotherapy, Parkinson disease.

\section{INTRODUÇÃO}

O tratamento fisioterapêutico de Parkinsonianos objetiva preservar as habilidades motoras e reduzir o avanço da doença e dos déficits motores, para melhorar o desempenho funcional $^{1}$.

A Doença de Parkinson (DP) é o distúrbio motor mais comum dos núcleos da base e caracteriza-se por acometimento de neurônios dopaminérgicos resultando em desordens dos movimentos voluntários e automáticos. A perda de neurônios dopaminérgicos e a redução da dopamina estriatal resultam nos sintomas de tremor, bradicinesia, rigidez e inabilidade postural $^{2}$.

Essa população apresenta uma marcha de velocidade lenta, com menor comprimento do passo, aumento compensatório na cadência, maior tempo da fase de duplo apoio e alta variabilidade na passada. A reabilitação em grupo auxilia na melhora do estado de saúde global do paciente e aumenta a socialização entre os indivíduos ${ }^{3}$.

O treinamento de parkinsonianos deve ser focado em prática de tarefas funcionais repetitivas, que podem melhorar o desempenho motor e a capacidade aeróbica. Programas terapêuticos que necessitam de agilidade sensório-motora vêm sendo aplicados na DP, destacando-se exercícios que envolvem coordenação, equilíbrio, destreza e treino de marcha com variação da velocidade, associação de tarefas cognitivas e utilização de sequências mais desafiadoras ${ }^{4,5}$.

A Fisioterapia em Grupo no formato de Circuit Training (FGCT) é um modelo de fisioterapia que utiliza exercícios funcionais específicos realizados de forma intensiva e difere da fisioterapia convencional devido a sua configuração de grupo e com foco na repetição e 
progressão contínua de exercícios em uma série de estações de trabalhos dispostos num formato de circuito dirigido, visando melhorar a mobilidade geral, a capacidade de andar, a coordenação e o equilíbrio funcional. Estudos demonstraram que a FGCT está associada a um maior grau de independência em diversas atividades e ainda a uma melhor satisfação no momento da alta da reabilitação do que a fisioterapia convencional individual $(\mathrm{FCl})^{6}$.

Sendo assim o objetivo deste trabalho é apresentar as atividades terapêuticas do Circuit Training desenvolvidas no grupo de Parkinsone e as características motoras funcionais dos parkinsonianos participantes.

\section{MÉTODOS}

Participaram deste estudo 14 parkinsonianos em atendimento em um Grupo de Atendimento Fisioterapêutico de Parkinsonianos (GAFP) submetidos à FGCT. Para participar deste estudo o paciente necessitou de diagnostico clínico de DP, estar entre o estágio 1 e 3 da escala de Hoehn e Yahr, ser capaz de realizar a marcha com ou sem auxílio de órtese, ter habilidade para realizar teste de caminhada e não apresentar déficits cognitivos avaliados pelo mini-exame do estado mental ${ }^{7,8}$. Todos os voluntários participantes foram informados sobre os objetivos e procedimentos do estudo e após concordarem com sua participação assinaram um termo de consentimento livre e esclarecido submetido ao Comitê de Ética da FCT (CAAE: 47863415.6.000.56402).

Antes de iniciar a FGCT os pacientes foram submetidos a uma entrevista individual para coleta de dados e a seguir foi realizada a avaliação inicial (AV1) utilizando as escalas de avaliação: 1) Lower Extremity Motor Coordination Test (LEMOCOT), que analisa a coordenação motora dos membros inferiores; 2 ) Time up and go (TUG), que avalia a mobilidade funcional e a marcha; 3) Teste de velocidade de marcha de 10 metros (TV10M), que avalia o tempo gasto para percorrer 10 metros; 4) Escala de Estágios de Incapacidade de Hoehn e Yahr, que avalia a incapacidade dos indivíduos com DP através dos seus sinais e sintomas classificandoos em estágios de 0 a 5 . Os pacientes participantes do grupo de Parkinson são avaliados semestralmente. Os resultados são tabulados e comparados entre si. Após serem submetidos à primeira avaliação os pacientes iniciaram seu tratamento na nova terapêutica ${ }^{7,9-}$ 11.

A FGCT ocorre no formato de estações, que corresponde a um determinado exercício, previamente montados em espaços diferentes e interligados. Os exercícios têm diferentes níveis de dificuldades, utilizando bastões, bolas, bambolês, cadeiras, escada e rampa, cones e mapas de sinalização. Cada paciente realiza o exercício na velocidade que consegue, porém é estimulado a realizar cada vez mais rápido para que o nível de dificuldade seja aumentado. Ao chegar à terapia, os terapeutas aferem a pressão arterial e cada paciente recebe um número correspondente à estação que ele deverá iniciar. Ele permanece por 2 minutos em cada estação e após este tempo, troca de estação. Durante a sessão, que tem duração de 60 minutos, ele passa por todas as estações, podendo ou não repetir algumas delas.

Antes de iniciar as estações, os pacientes realizam alongamentos globais, realizados de forma ativa, orientados por um fisioterapeuta.

As estações são: 1a - Quatro marcações de $10 \mathrm{~cm}$ de diâmetro, colocadas no chão formando um quadrado. $O$ paciente na posição ortostática segurando um bastão deve tocar as marcações, realizando rotação de tronco; 2a Três marcações colocadas na parede a $150 \mathrm{~cm}$ de altura do chão, formando um triângulo. $\mathrm{Na}$ posição ortostática, o paciente deve tocar na marcação e em seguida tocar seu nariz, alternando os braços; 3a - Na posição sentada, o paciente deve segurar uma bola de tamanho 10 com uma das mãos. Ele deve segurar a bola em uma das mãos e abduzir esse braço, em seguida encontra os braços na linha média, trocando a bola de mão, e abduzir o braço que está segurando a bola; 4a - Seis bambolês dispostos em pares, no chão. O paciente deve passar pelos bambolês colocando um pé dentro de cada bambolê, ao final caminha por fora dos bambolês retornando para o início; 5a - Três marcações de $10 \mathrm{~cm}$ de diâmetro posicionados em forma um triângulo, colados na parede a $220 \mathrm{~cm}$ do chão. $\mathrm{Na}$ posição ortostática e segurando um bastão com as mãos, o paciente deve tocar cada círculo com a outra extremidade do bastão; $6 \underline{a}$ - $\mathrm{Na}$ posição ortostática, o paciente segura uma bola tamanho $10 \mathrm{com}$ uma das mãos e passa a bola para a outra quando as mãos se encontrarem na frente e atrás do tronco, de modo que a bola contorne sua cintura; 7ạ - Em pé, apoiando em uma barra de ação, o paciente deve realizar 
agachamentos; 8a - Sentado em uma cadeira, próximo a uma mesa onde está uma caixa com pequenos blocos de madeira. $O$ paciente deve pegar um bloco e colocar do outro lado da caixa, alternando as mãos; 9a - Em uma escada rampa em forma de "L", com 5 degraus em um lado, uma plataforma plana de $(90 \times 90) \mathrm{cm}$ interligada com uma rampa de 6 graus de inclinação. 0 paciente deve subir pela escada e descer pela rampa e vice versa; 10 - Em um step retangular com altura de $15 \mathrm{~cm}$, posto no chão, onde 0 indivíduo deve realizar tríplice flexão com a perna, de modo a colocar alternadamente os pés sobre o mesmo. Estas sessões de FGCT ocorrem duas vezes por semana.

\section{RESULTADOS}

As avaliações iniciais demonstraram que os 14 parkinsonianos participantes apresentaram idade média de $72,53 \pm 6,44$ anos, variando de 61 a 81 anos. Destes, 13 são do gênero masculino e 1 do feminino. O tempo médio do aparecimento dos primeiros sinais e diagnóstico da doença foi de $6,33 \pm 5,34$ anos, variando de 8 meses a 20 anos. Estão classificados nos estágios $2 \pm 1$, segundo a Escala de Estágios de Incapacidade de Hoehn e Yahr, variando do estágio 0 a 3 .

A análise dos valores do LEMOCOT realizado pelos parkinsonianos deste estudo demonstrou uma média de 35,21 $\pm 12,01$ acertos nos alvos no lado dominante e $33,35 \pm 9,42$ no não dominante. $O$ tempo médio para percorrer a distancia no TV10M foi 9,73 $\pm 2,19$ seg e de 15,83 $\pm 8,95$ seg no TUG.

Este novo modelo terapêutico foi implantado em maio de 2015 e os pacientes apresentaram-se bastante motivados e satisfeitos com a terapia, pois participaram efetivamente durante as tarefas propostas.

Todo início da sessão de FGCT, é realizado a aferição de pressão arterial, seguido por alongamento global e distribuídos cartões com o número da estação em que cada paciente irá iniciar o tratamento. Esta distribuição ocorre para que haja uma melhor organização da dinâmica da sessão, além de estimular a cognição, a organização temporal e a noção espacial. Nas primeiras sessões pode-se observar que os pacientes apresentavam dificuldades de encontrar suas estações, além da dificuldade de execução adequada dos exercícios funcionais. Com o passar do tempo tornaram-se mais aptos, independentes e mais dinâmicos.
Apesar do trabalho ainda não apresentar dados que comprovem o seu benefício, a literatura tem demonstrado que esta terapêutica é eficaz além de ser uma alternativa no tratamento ambulatorial de parkinsonianos. Além disso, o tratamento proporciona uma interação social entre parkinsonianos e também pode ser interpretada como um estímulo durante a prática de exercícios funcionais.

\section{DISCUSSÃO}

O presente estudo é composto por parkinsonianos de ambos os gêneros, classificados em estágios de Hoehn e Yahr entre 0 ao 3 e que apresentam uma larga faixa de tempo de aparecimento dos primeiros sinais da doença ou de diagnóstico, variando de 8 meses a 20 anos. Pode-se observar que a progressão da doença, nesses indivíduos, não apresenta relação direta com o tempo de aparecimento dos primeiros sinais e de diagnóstico com o estágio no qual eles estão classificados, pois alguns pacientes com mais de 5 anos diagnosticados apresentam em estágios mais brandos de incapacidade enquanto pacientes com menos de 5 anos diagnosticados estão classificados em estágios mais avançados da incapacidade, segundo a escala de Hoehn e Yahr.

As desordens da marcha e do equilíbrio tem se apresentado como os principais objetivos terapêuticos nessa população. Nas fases iniciais da doença essas desordens são pouco incapacitantes, agravando-se com a progressão da mesma, que pode estar relacionada com a perda da eficácia do tratamento dopaminérgico ${ }^{12}$.

No presente estudo os pacientes apresentam coordenação motora dos membros inferiores próxima do esperado. Segundo os resultados do LEMOCOT o numero de acertos nos alvos encontram-se próximos dos valores preditos, para cada indivíduo, tanto do lado dominante como para o lado não dominante.

A perda da ritmicidade cortical foi correlacionada com piora da bradicinesia, contribuindo para uma menor adaptabilidade do comando motor a tarefas e consequentemente um déficit no recrutamento muscular capaz de iniciar o movimento em tempo adequado. Essas inabilidades puderam ser observadas no resultado do tempo gasto na realização do TUG. Neste teste o indivíduo deve levantar-se de uma cadeira, caminhar a distância de 3 metros em 
direção a um cone, dar a volta no cone, percorrer mais 3 metros em direção à cadeira e sentar-se novamente. Para realizar essa atividade os participantes gastaram um tempo médio de $15,83 \pm 8,95 \mathrm{seg}$, enquanto que no TVM10M percorreram a distância do teste em um tempo médio de 9,73 $\pm 2,19 \mathrm{seg}$, mesmo a distância sendo maior. Na DP a realização de movimentos simples que estejam integrados em uma sequência motora é caracterizado por pausas prolongadas, tais pausas foram observadas na realização do TUG, principalmente nas fases que havia presença de obstáculos, como o cone ou a cadeira. A lentidão na realização de movimentos, principalmente em atividades mais complexas simultâneas e sequenciais, são características da DP e foram percebidas na realização dos testes e nos resultados ${ }^{3,13,14}$

A reabilitação em grupo auxilia na melhora do estado de saúde global do paciente e aumenta a socialização entre os indivíduos. Programas terapêuticos que exploram a agilidade sensório-motora vêm sendo aplicada na DP, envolvendo atividades de coordenação, equilíbrio, destreza e treino de marcha com variação da velocidade, associação de tarefas cognitivas, realizadas de uma forma intensiva, com foco na repetição e progressão dos exercícios. Esse modelo fisioterapêutico tem como objetivo melhorar a mobilidade geral, a capacidade de andar, a coordenação e o equilíbrio funcional. Após a implantação do FGCT como um novo modelo terapêutico os pacientes apresentaram-se bastante motivados e satisfeitos com a terapia, pois participaram efetivamente durante as tarefas propostas, além de proporcionar maior interação entre os participantes $^{1,4}$.

\section{CONCLUSÃO}

A experiência com a FGCT permite afirmar que é uma terapia segura, viável e aplicável em parkinsonianos e inferir que estes pacientes tenham um espaço terapêutico diferente que os permite exercitarem e se adaptarem às suas limitações, evitando a progressão das suas incapacidades e sedentarismo.

\section{CONFLITO DE INTERESSE}

Os autores declaram não haver qualquer potencial de conflito de interesse que possa interferir na imparcialidade deste trabalho científico.

\section{REFERÊNCIAS}

1. Tomlinson CL, Patel S, Meek C, Herd CP, Clarke CE, Stowe $\mathrm{R}$ et al. Physiotherapy intervention in Parkinson's disease: systematic review and metaanalysis. $\mathrm{Br}$ Med J. 2012;345:e5004. DOI: http://dx.doi.org/10.1136/bmj.e5004

2. Hallett M. Parkinson revisited: pathophysiology of motor signs. Advances in neurology. 2003;91:19-28.

3. Giladi N. Mobility and exercise in movement disorders. Parkinsonism Relat Disord. 2009;15(Suppl 3):S46-8. DOI: http://dx.doi.org/10.1016/S13538020(09)70779-5

4. de Dreu MJ, van der Wilk AS, Poppe E, Kwakkel G, van Wegen EE. Rehabilitation, exercise therapy and music in patients with Parkinson's disease: a metaanalysis of the effects of music-based movement therapy on walking ability, balance and quality of life. Parkinsonism Relat Disord. 2012;18(Suppl 1):S114-9. DOI: http://dx.doi.org/10.1016/S1353-8020(11)70036$\underline{0}$

5. Allen NE, Sherrington C, Suriyarachchi GD, Paul SS, Song J, Canning CG. Exercise and motor training in people with Parkinson's disease: a systematic review of participant characteristics, intervention delivery, retention rates, adherence, and adverse events in clinical trials. Parkinson's disease. 2012;2012:854328. DOI: http://dx.doi.org/10.1155/2012/854328

6. English CK, Hillier SL, Stiller KR, Warden-Flood A. Circuit class therapy versus individual physiotherapy sessions during inpatient stroke rehabilitation: a controlled trial. Arch Phys Med Rehab. 2007;88(8):955-63.

DOI:

http://dx.doi.org/10.1016/i.apmr.2007.04.010

7. Hoehn MM, Yahr MD. Parkinsonism: onset, progression, and mortality. 1967. Neurology. 2001;57(10 Suppl 3):S11-26.

8. Cockrell JR, Folstein MF. Mini-Mental State Examination (MMSE). Psychopharmacol Bull. 1988;24(4):689-92.

9. Pinheiro MB, Scianni AA, Ada L, Faria CD, TeixeiraSalmela LF. Reference values and psychometric properties of the lower extremity motor coordination test. Arch Phys Med Rehab. 2014;95(8):1490-7. DOI: http://dx.doi.org/10.1016/j.apmr.2014.03.006

10. Podsiadlo D, Richardson S. The timed "Up \& Go": a test of basic functional mobility for frail elderly persons. J Am Geriat Soc. 1991;39(2):142-8. DOI: http://dx.doi.org/10.1111/i.1532-

\subsection{1.tb01616.x}

11. Bowden JD, Bauerle WL. Measuring and modeling the variation in species-specific transpiration in temperate deciduous hardwoods. Tree Physiol. 2008;28(11):1675-83.

DOI: 
12. Grabli D, Karachi C, Welter ML, Lau B, Hirsch EC, Vidailhet $\mathrm{M}$, et al. Normal and pathological gait: what we learn from Parkinson's disease. J Neurol, Neurosurg Psych. 2012;83(10):979-85. DOI: http://dx.doi.org/10.1136/innp-2012-302263

13. Berardelli A, Rothwell JC, Thompson PD, Hallett M. Pathophysiology of bradykinesia in Parkinson's disease. Brain J Neurol. 2001;124(Pt 11):2131-46.

14. Calne D. Parkinsonism and Related Disorders: Looking back over twenty years. Parkinsonism Relat Disord. 2015;21(9):1011-2. DOI: http://dx.doi.org/10.1016/j.parkreldis.2015.05.026

Recebido para publicação em 28/08/2015

Revisado em 08/09/2015

Aceito em 12/09/2016 